\begin{tabular}{|c|c|c|}
\hline \multirow{3}{*}{$\begin{array}{r}\text { Case Reports in } \\
\text { Gastroenterology }\end{array}$} & \multirow{2}{*}{\multicolumn{2}{|c|}{ Case Rep Gastroenterol 2015;9:36-42 }} \\
\hline & & \\
\hline & $\begin{array}{l}\text { DOI: 10.1159/000375116 } \\
\text { Published onlIne: January 27, } 2015\end{array}$ & $\begin{array}{l}\text { (c) } 2015 \text { S. Karger AG, Basel } \\
1662-0631 / 15 / 0091-0036 \$ 39.50 / 0 \\
\text { www.karger.com/crg }\end{array}$ \\
\hline & \multicolumn{2}{|c|}{$\begin{array}{l}\text { This is an Open Access article licensed under the terms of the Creative Commons } \\
\text { Attribution-NonCommercial } 3.0 \text { Unported license (CC BY-NC) (www.karger.com/OA- } \\
\text { license), applicable to the online version of the article only. Distribution permitted for non- } \\
\text { commercial purposes only. }\end{array}$} \\
\hline
\end{tabular}
commercial purposes only

\section{Nitazoxanide Use as Part of an Empiric Multi-Drug Regimen in Treating Children with Suspected Helicobacter pylori Infection}

\author{
Asuncion G. Ramos-Soriano ${ }^{a}$ Jimmy Black ${ }^{b}$ \\ ${ }^{a}$ Laredo Medical Center, Laredo, Tex., and ${ }^{b}$ Atom Strategic Consulting, LLC, \\ Morristown, N.J., USA
}

\section{Key Words}

Nitazoxanide $\cdot$ Multi-drug regimen $\cdot$ Children $\cdot$ Helicobacter pylori infection · Gastritis

\begin{abstract}
Helicobacter pylori, a Gram-negative bacterium found in the human stomach, is often present in patients with chronic gastritis. Traditional treatment for $\mathrm{H}$. pylori infection includes metronidazole or clarithromycin, both being associated with development of resistance. In this retrospective report, we describe our clinical experience using a multi-drug treatment regimen for pediatric $H$. pylori that included nitazoxanide, a newer nitrothiazole benzamide compound used in treating intestinal protozoa infections. Charts were identified for patients who were treated between January 1, 2008 and December 31, 2013 with an ICD-9-CM code 041.86 (H. pylori) and who underwent elective endoscopy. All patients were exposed to nitazoxanide for 3 days plus azithromycin, and cefixime (or another 3rd-generation oral cephalosporin) for 7-10 days, plus a proton pump inhibitor for 30 days. The clinical cure criteria were predefined. There were 127 individual occurrences or cases identified for inclusion in the review, with 111 occurrences meeting the inclusion criteria. The success rate or clinical cure for the new therapy combination prescribed as defined prior to the chart review was 99 out of 111 cases (89.2\%). There were no serious adverse events observed or reported during the treatment of any patient. Approximately $10 \%$ of patient charts reflected minor complaints of nausea, vomiting or abdominal cramps during the time of active drug therapy. Nitazoxanide appears to be an effective and well-tolerated option for use in combination with other agents to treat $H$. pylori-induced gastritis.

(c) 2015 S. Karger AG, Basel
\end{abstract}


Ramos-Soriano and Black: Nitazoxanide Use as Part of an Empiric Multi-Drug Regimen in Treating Children with Suspected Helicobacter pylori Infection

\section{Introduction}

Helicobacter pylori is a Gram-negative bacterium found in the human stomach. It was identified in the early eighties by Australian scientists Barry Marshall and Robin Warren, who found that it was present in patients with chronic gastritis and gastric ulcers, conditions that were not previously believed to have a microbial cause. It is also linked to the development of duodenal ulcers and gastric cancer. However, over $80 \%$ of individuals infected with the bacterium are asymptomatic, and it has been postulated that it may play an important role in the natural stomach ecology [1].

The traditional first-line treatment regimens for $H$. pylori often include metronidazole or clarithromycin as the backbone of antibiotic therapy. Unfortunately, metronidazole and clarithromycin are associated with significant patterns of resistance, with in vitro resistance to these antibiotics being reported as high as 40 and 13\%, respectively [2, 3]. Nitazoxanide is a newer nitrothiazole benzamide compound notable for its activity in treating both intestinal protozoa and helminthic infections, with a low range of minor adverse effects [4].

Nitazoxanide has been reported to be effective against a broad range of parasites, including Giardia lamblia, Entamoeba histolytica, Cryptosporidium parvum and Ascaris lumbricoides [5]. The antiprotozoal activity of nitazoxanide is believed to be due to interference with the pyruvate:ferredoxin oxidoreductase (PFOR) enzyme-dependent electron transfer reaction, which is essential to anaerobic energy metabolism [6]. The US Food and Drug Administration (FDA) approved the oral suspension of nitazoxanide in December 2002 for the treatment of diarrhea caused by Cryptosporidium spp. and G. lamblia in pediatric patients. The first research on the antiviral effects of nitazoxanide was published in $2006[7,8]$. Tizoxanide (the active metabolite of nitazoxanide) showed a cytoprotective effect in rotavirus-infected cells in studies carried out in vitro [7]. Double-blind, placebo-controlled trials in children and adults have shown a significant reduction in the median duration of diarrhea from the first dose of nitazoxanide administration in comparison with placebo [7, 8]. With the increased resistance and high prevalence of $H$. pylori infection in South Texas, an effective alternative regimen to the standard of care is warranted and desirable. The purpose of this review is to report our clinical experience using an alternative multi-drug therapy for the treatment of pediatric $H$. pylori infection.

\section{Methods}

\section{Study Design and Population}

Charts were identified for patients who were treated between January 1, 2008 and December 31, 2013 with an ICD-9-CM code 041.86 (H. pylori) and who underwent elective endoscopy. Patients included in the review were described to suffer from the following clinical symptoms: chronic abdominal pain; chronic nausea or vomiting, chronic gastroesophageal reflux disease; chronic diarrhea; persistent bloating or flatulence; anal pruritus; nocturnal teeth grinding; gross or occult intestinal bleeding; and most importantly feeding problems with or without weight loss. Chronic as defined clinically is usually greater than 1 or 2 months of symptoms (American Academy of Pediatrics). All patients included in the chart review were treated with a combination of nitazoxanide twice daily for 3 days and a 3rdgeneration cephalosporin antibiotic (cefixime in the majority) once daily and a macrolide antibiotic (azithromycin) once daily for 7-10 days plus a proton pump inhibitor (PPI) once daily for 30 days. Clinical cure was defined as a negative urea breath test with resolution of symptoms on the subsequent visit at no more than 4 months following antimicrobial drug 
Ramos-Soriano and Black: Nitazoxanide Use as Part of an Empiric Multi-Drug Regimen in Treating Children with Suspected Helicobacter pylori Infection

therapy initiation. Patients without follow-up urea breath test were evaluated for clinical response and resolution of presentation symptoms.

Patients excluded from the analysis were those who failed to return for follow-up or had incomplete information in the chart. Exclusion criteria consisted of persistence or recurrence of infection within 4 months of initiation of the original drug therapy.

\section{Definitions}

Clinical cure was assessed no greater than 4 months after initiation of the aforementioned combination drug therapy. Clinical cure was determined to have been achieved if the patient was free from all inclusion criteria signs and symptoms as described in the methodology. Moreover, to avoid confounding medical problems, patients had to not be receiving any course of antibiotics for any other medical condition. Failure to achieve any of these endpoints was by definition clinical failure.

\section{Assessment}

All patients included in the review underwent an elective esophagogastroduodenoscopy endoscopic evaluation during which tissue samples were obtained for evaluation, which included histological determination of $H$. pylori infection. Visual assessment of the characteristic $H$. pylori nodularity and erosive or ulcer disease warranted empiric therapy pending histopathology confirmation.

\section{Results}

There were 112 charts identified for the period in question that represented 127 individual occurrences for evaluation in the review. Of these, 111 occurrences met all of the criteria for inclusion in the review. Table 1 shows the simple demographics for the patients included in this review. The clinical success rate for this series of patients using the new drug regimen as described is shown in table 2 . The success rate for the new therapy combination prescribed as defined prior to the review was 99 out of 111 cases (89.2\%). The drug therapy assessed consisted of nitazoxanide, azithromycin and cefixime. In six cases and one case respectively, ceftibuten and cefdinir were substituted for cefixime. Table 3 shows the dosing used for the 111 cases reported. Nitazoxanide suspension $(100 \mathrm{mg} / 5 \mathrm{ml})$ was used in 66 cases $(59.5 \%)$ while the $500 \mathrm{mg}$ tablet was prescribed for the remaining 45 patients $(40.5 \%)$.

There were no serious adverse events observed or reported during the treatment course for any patient. Approximately $10 \%$ of patient charts to some degree reflected a complaint of nausea, vomiting or abdominal cramps during the time of active drug therapy. The charts for the twelve cases defined as clinical failures all reflected persistence of mild to moderate abdominal pain, nausea and/or vomiting during the entire post-treatment course up to and beyond 4 months. There was a single case of facial rash reported in one patient who was among the twelve characterized as clinical failures. As the drug regimen used for this series of patients included four drugs, it was difficult to attribute causality to any single agent.

\section{Limitation}

Endoscopic visual confirmation of suspected H. pylori (via Olympus esophagogastroduodenoscopy endoscope with HDTV; colored photos attached to patient chart) with peptic erosive or ulcer disease was relied upon for prompt treatment in the light of the patients' 
Ramos-Soriano and Black: Nitazoxanide Use as Part of an Empiric Multi-Drug Regimen in Treating Children with Suspected Helicobacter pylori Infection

chronic gastrointestinal signs and symptoms and risk factors noted in the clinical history. In addition, in community-based clinical practice, systematic protocols require more extensive planning with trained personnel. However, despite the inherent limitations of this retrospective review, analysis of data demonstrated significant clinical improvement within a significant period of time post diagnosis and initiation of treatment.

\section{Discussion}

H. pylori is a Gram-negative, spiral bacterium situated on the epithelial surface of the stomach [6]. It is thought to be the most common bacterial infection worldwide [9]. Virtually all persons infected by this organism develop gastritis, a signature feature of which is the capacity to persist for decades, leading to chronic inflammation of the underlying mucosa [6]. The organism can resist the harsh acidic environment of the stomach due to its high urease activity; urease converts the urea present in gastric juice to alkaline ammonia and carbon dioxide, thereby raising the $\mathrm{pH}$ of the stomach and allowing it to thrive [6]. In chronic disease states as well as in high-risk and immunocompromised populations, $H$. pylori infection leads to peptic ulcer and gastric cancer [6].

In the past, drug regimens that included a PPIs in combination with several antibiotics such as clarithromycin, amoxicillin and metronidazole given 7-14 days in divided doses were very successful for $H$. pylori eradication $[10,11]$. Unfortunately, there are reports of reduced efficacy of these combinations as a result of the emerging problem of antibiotic resistance both in developing and developed countries [12]. This has led to the use of newer and arguably more powerful antibiotic combinations that include tetracycline, doxycycline, clarithromycin and levofloxacin, to name a few.

The use of nitazoxanide in the treatment of $H$. pylori as part of a multi-drug regimen has been described by others [13]. An investigational four-drug regimen for the ulcer-causing H. pylori infection led to a significantly higher eradication rate than the current standard in previously untreated patients in a multi-center, randomized trial reported in 2009 during the Annual Meeting of the American College of Gastroenterology. The new four-drug treatment, known as LOAD, comprises levofloxacin, omeprazole (Prilosec), nitazoxanide (Alinia) and doxycycline. It was tried against the normal three-drug regimen, known as LAC, which stands for lansoprazole (Kapidex, Prevacid), amoxicillin and clarithromycin. Seven-day and 10 -day courses of the LOAD regimen led to eradication rates of about $90 \%$, compared with $75 \%$ in patients treated with the LAC regimen. Adverse event rates were similar, except for abdominal pain, which occurred significantly less often under the LOAD regimen. The utility of nitazoxanide as monotherapy in the treatment of $H$. pylori gastritis has also been described by others, but is not the focus of our review and so will not be addressed here.

In this report, a pediatric clinical experience of empiric use of nitazoxanide (for suspected metronidazole-resistant strains) in combination with a 3rd-generation cephalosporin (for suspected amoxicillin resistance), azithromycin (for suspected clarithromycin resistance) and a PPI were prescribed to manage $H$. pylori peptic ulcer and erosive disease post endoscopy. Nitazoxanide, a nitrothiazolyl-salicylamide compound, was first described by Rossignol and Cavier [14]. The selection of nitazoxanide was based on its reported broad spectrum of activity against microaerobic and anaerobic bacteria, anaerobic protozoa and helminths $[15,16]$. Alinia ${ }^{\circledR}$ tablets and Alinia ${ }^{\circledR}$ oral suspension contain the active ingredient nitazoxanide, a synthetic antiprotozoal agent for oral administration [17]. Following oral administration of nitazoxanide, maximum plasma concentrations of the active metabolites tizoxanide and tizoxanide glucuronide are observed within 1-4 h [17]. The parent nitazoxa- 
Ramos-Soriano and Black: Nitazoxanide Use as Part of an Empiric Multi-Drug Regimen in Treating Children with Suspected Helicobacter pylori Infection

nide is not detected in plasma [17]. Following oral administration in humans, nitazoxanide is rapidly hydrolyzed to an active metabolite, tizoxanide (desacetyl-nitazoxanide) [17]. Tizoxanide then undergoes conjugation, primarily by glucuronidation [17]. In vitro metabolism studies have demonstrated that tizoxanide has no significant inhibitory effect on cytochrome P450 enzymes [17]. Tizoxanide is excreted in urine, bile and feces, and tizoxanide glucuronide is excreted in urine and bile [17]. Approximately two-thirds of the oral dose of nitazoxanide is excreted in the feces and one-third in the urine [17].

The antiprotozoal activity of nitazoxanide is believed to be due to interference with the PFOR enzyme-dependent electron transfer reaction in a unique non-competitive binding by targeting thiamine pyrophosphate (cofactor to PFOR), which disrupts pyruvate oxidation and energy production, leading to death of the organism [17]. This mechanism of action is postulated to avoid mutation-based drug resistance [17]. Nitazoxanide also disrupts two other enzyme systems: nitroreductases and protein disulfide isomerases, which are minor but essential to anaerobic energy metabolism [18]. These inhibitory actions may account for its activity against $H$. pylori infection [18].

Nitazoxanide oral suspension (for patients 1 year of age and older) and tablets (for patients 12 years and older) are indicated for the treatment of diarrhea caused by G. lamblia or C. parvum. In vitro, nitazoxanide has activity against anaerobic bacteria (Bacteroides spp. and Clostridium spp.) and some aerobic Gram-positive bacteria and viruses [17]. The standard dosing frequency of nitazoxanide is every $12 \mathrm{~h}$ with food for 3 days [17]. It is the short 3-day course of nitazoxanide, coupled with its very favorable safety profile, that makes the drug attractive for empiric drug therapy for the treatment of $H$. pylori.

This retrospective study demonstrates a clinical success similar to that reported by Basu et al. [13] for the LOAD protocol (89 vs. 90\%, respectively). It describes clinical success in the use of a combination regimen of nitazoxanide, azithromycin, a 3rd-generation cephalosporin, and a PPI in the treatment of endoscopically diagnosed H. pylori peptic ulcer or erosive disease in chronically symptomatic children. Unlike the LOAD protocol, the regimen of nitazoxanide used was and is consistent with the current label of twice daily administration for 3 days. The drug is well tolerated and use of the liquid formulation in children offers the advantage of easy, well-accepted administration of a pleasant-flavored liquid.

Nitazoxanide in the treatment of $H$. pylori is not an approved indication yet. However, the clinical experience described appears to support the synergistic action of nitazoxanide with other antibiotics, especially in clinically suspected multi-drug resistance to metronidazole, amoxicillin and clarithromycin. It is well tolerated, with the most common adverse events reported regardless of causality assessment being abdominal pain (6.6\%), diarrhea $(4.2 \%)$, headache $(3.1 \%)$ and nausea $(3.0 \%)$. The oral suspension formulation of nitazoxanide in a pink pleasant strawberry flavor presents a more palatable administration especially in children.

This clinical experience presents a viable treatment option in community-based clinical settings where multiple drug-resistant strains of $H$. pylori are suspected in a high-risk population. The ongoing emergence of multiple drug-resistant organisms is a serious public health dilemma. The results of this study suggest an alternative regimen that can readily be initiated in cases where immediate treatment is a priority, pending further diagnostic studies. In the pediatric population where chronic gastrointestinal disease produces profound feeding and nutritional deficiencies, initiation of therapy in the interest of preventing and reversing immune deficiency is of critical importance.

Despite the inherent limitations of this retrospective study, the efficacy of a 3-day course of nitazoxanide is worth additional investigation to clearly determine its efficacy in the treatment of $H$. pylori peptic ulcer disease and prevention of gastric cancer. 
Ramos-Soriano and Black: Nitazoxanide Use as Part of an Empiric Multi-Drug Regimen in Treating Children with Suspected Helicobacter pylori Infection

\section{Disclosure Statement}

The authors have no relevant financial relationships to disclose.

\section{References}

1 Blaser MJ: Who are we? Indigenous microbes and the ecology of human diseases. EMBO Rep 2006; 7: 956-960.

-2 Osato MS, Reddy R, Reddy SG, Penland RL, Malaty HM, Graham DY: Pattern of primary resistance of Helicobacter pylori to metronidazole or clarithromycin in the United States. Arch Intern Med 2001;161: 1217-1220.

-3 Mégraud F: H. pylori antibiotic resistance: prevalence, importance, and advances in testing. Gut 2004;53: 1374-1384.

-4 Parashar UD, Hummelman EG, Bresee JS, Miller MA, Glass RI: Global illness and deaths caused by rotavirus disease in children. Emerg Infect Dis 2003;9:565-572.

5 Szajewska H, Mrukowicz JZ: Probiotics in the treatment and prevention of acute infectious diarrhea in infants and children: a systematic review of published randomized, double-blind, placebo-controlled trials. J Pediatr Gastroenterol Nutr 2001;33(suppl 2):S17-S25.

-6 McColl KE: Clinical practice. Helicobacter pylori infection. N Engl J Med 2010;362:1597-1604.

7 Rossignol JF, Abu-Zekry M, Hussein A, Santoro MG: Effect of nitazoxanide for treatment of severe rotavirus diarrhoea: randomised double-blind placebo-controlled trial. Lancet 2006;368:124-129.

-8 Rossignol JF, El-Gohary YM: Nitazoxanide in the treatment of viral gastroenteritis: a randomized doubleblind placebo-controlled clinical trial. Aliment Pharmacol Ther 2006;24:1423-1430.

-9 Francois F, Blaser MJ: Improving Helicobacter pylori eradication regimens. Ann Intern Med 2006;144: $140-141$.

10 Gisbert JP, González L, Calvet X, García N, López T, Roqué M, Gabriel R, Pajares JM: Proton pump inhibitor, clarithromycin and either amoxycillin or nitroimidazole: a meta-analysis of eradication of Helicobacter pylori. Aliment Pharmacol Ther 2000;14:1319-1328.

11 Laine L, Fennerty MB, Osato M, Sugg J, Suchower L, Probst P, Levine JG: Esomeprazole-based Helicobacter pylori eradication therapy and the effect of antibiotic resistance: results of three US multicenter, doubleblind trials. Am J Gastroenterol 2000;95:3393-3398.

12 Buta N, Tanih NF, Ndip RN: Increasing trend of Metronidazole resistance in the treatment of Helicobacter pylori infection. A global challenge. Afr J Biotechnol 2010;9:1115-1121.

-13 Basu PP, Rayapudi K, Pacana T, Shah NJ, Krishnaswamy N, Flynn M: A randomized study comparing levofloxacin, omeprazole, nitazoxanide, and doxycycline versus triple therapy for the eradication of Helicobacter pylori. Am J Gastroenterol 2011;106:1970-1975.

14 Rossignol JF, Cavier R: A new derivative of 2-benzamido 5-nitrothiazoles. Chem Abstr 1975;83:28216.

15 Arya SC: Nitazoxanide as a broad-spectrum antiparasitic agent. J Infect Dis 2002;185:1692.

16 Dubreuil L, Houcke I, Mouton Y, Rossignol JF: In vitro evaluation of activities of nitazoxanide and tizoxanide against anaerobes and aerobic organisms. Antimicrob Agents Chemother 1996;40:2266-2270.

17 Alinia Prescribing Information. Romark Pharmaceuticals, June 2005.

18 Siddiq DM, Koo HL, Adachi JA, Viola GM: Norovirus gastroenteritis successfully treated with nitazoxanide. J Infect 2011;63:394-397.

Table 1. Patient demographics

\begin{tabular}{ll}
\hline Male & $65(58.6 \%)$ \\
Female & $46(41.4 \%)$ \\
\hline Age range, years & $1-21$ \\
Average age, years & 10.04 \\
\hline
\end{tabular}


Ramos-Soriano and Black: Nitazoxanide Use as Part of an Empiric Multi-Drug Regimen in Treating Children with Suspected Helicobacter pylori Infection

Table 2. Response rate to multi-drug therapy

\begin{tabular}{lr}
\hline Patients & $111(100 \%)$ \\
Clinical cure & $99(89.2 \%)$ \\
Clinical failure & $12(10.8 \%)$ \\
\hline
\end{tabular}

Table 3. Antibiotic therapy selection and distribution

\begin{tabular}{lllll}
\hline & Frequency & $\begin{array}{c}\text { Average } \\
\text { dose, mg }\end{array}$ & $\begin{array}{l}\text { Duration, } \\
\text { days }\end{array}$ & $\begin{array}{l}\text { Number } \\
\text { received }\end{array}$ \\
\hline $\begin{array}{l}\text { Nitazoxanide } \\
\text { Azithromycin }\end{array}$ & $\begin{array}{l}\text { twice daily } \\
\text { once daily }\end{array}$ & 327.5 & 3 & 111 \\
+ & 215.1 & 10 & 111 \\
Cefixime & once daily & 278.8 & 10 & 104 \\
$\begin{array}{l}\text { Ceftibuten } \\
\text { Cefdinir }\end{array}$ & once daily & 246.0 & 10 & 6 \\
\hline
\end{tabular}

